\title{
VALUE OF PREOPERATIVE RADIOGRAPHIC EVALUATIONS ON KNEE BONE DEFECTS FOR REVISION ARTHROPLASTY
}

Mauricio Masasi lamaguchi' ${ }^{1}$ Camilo Partezani Helito², Riccardo Gomes Gobbi ${ }^{3}$, Marco Kawamura Demange ${ }^{3}$, Luiz Eduardo Passarelli Tirico', Jose Ricardo Pecora ${ }^{4}$, Gilberto Luis Camanho

\begin{abstract}
Objective: To evaluate the value of preoperative radiographic evaluations for total knee arthroplasty (TKA) revision. Methods: Thirty-one knees that were operated between 2006 and 2008 , in a consecutive series of cases of TKA revision surgery were analyzed retrospectively. The following criteria were evaluated: number of wedges or structured bone grafts used for filling the bone defects; locations of the wedges and bone grafts used; and mean thickness of the polyethylene used. The AORI classification was previously established based on preoperative radiographs, using preestablished criteria. After the analysis, the knees were divided into four groups (I, IIA, IIB and III). Results: The mean number of wedges or grafts used in each knee
\end{abstract}

progressively increased among the groups (group I: 1.33; group IIA: 2; group IIB: 4.33; and group III: 4.83$)(\mathrm{P}=0.0012)$. The commonest locations were medial in the tibia and posteromedial in the femur. There were no statistically significant differences in the thickness of the polyethylene used. Conclusion: The AORI classification for bone defects in the knee, based on preoperative radiographs, showed a correlation with increasing need to use wedges and/or structured grafts in TKA revisions. However, up to $46 \%$ of the knees in groups I and IIA presented bone defects of up to $5 \mathrm{~mm}$ that were not diagnosed by means of preoperative radiographs.

Keywords - Bone Defects; Total Knee Arthroplasty Revision/ classification; Radiography/classification

\section{INTRODUCTION}

Total knee arthroplasty is a surgical procedure that is becoming increasingly common ${ }^{(1,2)}$. Despite the gradual development of implants, an increasing number of total knee arthroplasty revision surgeries are becoming necessary.

Revision total knee arthroplasty is always a challenging surgical procedure, with less satisfactory clinical results than primary total knee arthroplasty ${ }^{(3,4)}$. The adequate planning of the surgery to be performed is, without doubt, the most important step in this procedure. This planning includes various factors to be analyzed, such as: data from the previous surgery, reason for the revision total knee arthroplasty (septic or aseptic), physical examination, and adequate radiographic assessment of the patient, among other factors.

There is a wide range of options of implant materials. Besides the implants used in primary total knee arthroplasties, there is also a wide range of components for semiconstricted implants available for revisions: stems of various sizes and diameters for the femur and tibia; various sizes of polyethylene; offset mechanisms to correct deformities between the center of the diaphysis and the center of the femoral or tibial joint; wedges of various sizes, shapes and angles, and made-to-measure modules for large tibial bone

1 - Orthopedist and Preceptor, Knee Group, Institute of Orthopedics and Traumatology, HC/FMUSP - São Paulo, Brazil.

2 - Resident, Knee Group, Institute of Orthopedics and Traumatology, HC/FMUSP - São Paulo, Brazil.

3 - Assistant Physician, Knee Group, Institute of Orthopedics and Traumatology, HC/FMUSP - São Paulo, Brazil.

4 - Assistant Physician and Head, Knee Group, Institute of Orthopedics and Traumatology, HC/FMUSP - São Paulo, Brazil

5 - Full Professor, Department of Orthopedics, USP School of Medicine - São Paulo, Brazil.

Study conducted at the Musculoskeletal System Medical Investigation Laboratory - LIM41 of the Department of Orthopedics and Traumatology of FMUSP.

Correspondence: Camilo Partezani Helito - Rua Dr. Ovídio Pires de Campos, 333, Cerqueira César - 05403-010 - São Paulo, SP. Email: Camilo_helito@yahoo.com.br Received for publication: 3/25/2011, accepted for publication: $1 / 11 / 2012$.

The authors declare that there was no conflict of interest in conducting this work 
losses ${ }^{(5-15)}$. And for cases of large bone and ligament loss, there are constricted implants ${ }^{(16-18)}$.

The choice of implant to be used is not always simple, and depends largely on three factors: quantification of the bone loss, assessment of the ligament deficiency, and function of the extensor mechanism. Evaluation of ligament deficiencies and of the function of the extensor mechanism of the knee is generally based on data obtained in the physical examination, information from previous surgeries, and imaging exams.

Dorr $^{(8)}$, Insall ${ }^{(19)}$, Rand ${ }^{(13)}$ and Engh ${ }^{(20,21)}$ are some authors of classification systems for assessing bone loss in the knee. A major challenge is to assess and quantify this loss, as the presence of the metal implant hinders radiographic interpretation, even for experienced surgeons.

Engh $^{(20,21)}$ describes a classification system for bone defects in the knee developed at the Anderson Orthopaedic Research Institute (AORI). This classification system is based on the findings after removing the components of total knee arthroplasty ${ }^{(20)}$, but it can also be estimated by means of preoperative radiographs, with a moderate correlation between surgeons $^{(20,22)}$. This classification system is widely used among knee surgeons, and is considered one of the best available options for this type of assessment. A score is attributed to the femur, and another is attributed to the tibia, as follows:

Type I: Preserved metaphysis bone with minimum bone defects, which do not alter the stability of the implant.

Type II: The damaged metaphysis bone subdivided into A (lesion of one femoral condyle or one tibial plateau) and B (lesion of both femoral condyles or tibial plateaux).

Type III: Extensive bone defect affecting a large part of the femoral condyle or tibial plateau, occasionally associated with a ligament deinsertion and instability of the collateral ligaments.

There have been few published works evaluating the importance and functionality of these classification systems. The aim of this work was to analyze the Anderson Orthopaedic Research Institute classification system for bone defects in the knee $\mathrm{e}^{(20,21)}$ through preoperative radiographs, and to evaluate its capacity to predict the need for the use of compensation for bone defects during surgery, whether by blocks, wedges, or bone grafts in revision total knee arthroplasty.

\section{MATERIALS AND METHODS}

Thirty-one knees (30 patients) operated on in the period between 2006 and 2008 in a consecutive case series of total knee arthroplasty revision surgeries were analyzed retrospectively, while following a prospective sequence of events, in which the AORI classification was first defined by preoperative radiographies, and then the data were compared and analyzed after the intraoperative findings. In all the patients, an implant with uncemented stem Scorpio ${ }^{\circledR}$ TS Total Knee Revision System (Stryker ${ }^{\circledR}$ ) was used. There were 18 women and 12 men, with a mean age of 69 years (45 to 82 years). The reasons for the revision surgery were aseptic loosening in 16 patients, septic loosening with two-stage revision in nine patients, and septic loosening with single-stage revision in six patients.

The bone defects were classified prior to surgery, based on preoperative radiographies in the front, true profile and axial projections of the patella, and panoramic projection of the lower limbs. The Anderson Orthopaedic Research Institute (AORI) bone defect classification system was used for femoral and tibial bone defects (Chart 1). In 22 knees this analysis was carried out based on radiographs in which the implant was present; 21 total knee arthroplasties, and one unicompartmental knee arthroplasty. In nine patients, this analysis was based on radiographies in which an orthopedic knee cement spacer was present. This initial classification was carried out by independent evaluators from those responsible for analyzing the intraoperative findings.

The patients were divided into four groups, according to the worst score obtained, whether in the femur or the tibia: group III (patients F3 and/or T3); group IIB (patients F2b and/or T2b who did not belong to group III); group IIA (patients F2a and/or T2a who did not belong to groups III or IIB); and group I (patients F1 and T1 exclusively) (Chart 2). The decision to use the score for the worst defect was a practical one, since the ability to predict, prior to the surgery, the worst case scenario that might be found intraoperatively is more important than absolute precision in the preoperative assessment. 
Records of intraoperative use of wedges to fill bone defects were analyzed and compared with the AORI score obtained prior to the surgery. The thickness of the tibial polyethylene was also recorded. The choice of the use of wedges, bone and polyethylene thickness to fill the bone defects found was not standardized in this study, since its scope is primarily to evaluate whether the AORI classification enables defects to be predicted before surgery, and the possible scoring difficulties, and not to evaluate the different techniques used for bone filling.

The statistical analysis of the number of wedges and the thickness of the polyethylene was performed by the Kruskal-Wallis test.

Chart 1 - Classification of bone defects in the femur and tibia (AORI).

\begin{tabular}{|c|c|}
\hline F1 & No bone defects, implant with adequate alignment \\
\hline F2a & Unicondylar bone defect or misalignment \\
\hline F2b & Bicondylar bone defect \\
\hline F3 & Bone defect involving one or both condyles \\
\hline T1 & No bone defects, implant with adequate alignment \\
\hline T2a & Unicondylar bone defect or misalignment \\
\hline T2b & Bicondylar bone defect \\
\hline T3 & Bone defects with extension below the fibular head \\
\hline
\end{tabular}

Chart 2 - Classification of the groups based on the worst AORI score.

\begin{tabular}{|c|c|}
\hline Groups & Preoperative radiograph \\
\hline III & F3 and/or T3 \\
\hline IIB & F2b and/or T2b \\
\hline IIA & F2a and/or T2a \\
\hline I & F1 and T1 \\
\hline
\end{tabular}

\section{RESULTS}

Thirty-one knees (30 patients) were analyzed, with six knees in group I, seven knees in group IIA, 12 knees in group IIB, and six knees in group III.

The most commonly used wedges or grafts were, in descending order: medial tibia, posteromedial femur, posterolateral femur, distal medial femur, distal lateral femur, lateral tibia, medial femoral autograft, and lateral tibial autograft (Table 1).

The use of wedges and the size of the polyethylene are shown in Table 2.
Table 1 - Wedges and grafts used.

\begin{tabular}{|c|c|c|c|}
\hline Femur & & Number of patients & $\%$ \\
\hline \multicolumn{2}{|c|}{ Posterolateral } & 17 & $54.84 \%$ \\
\hline \multicolumn{2}{|c|}{ Posteromedial } & 18 & $58.06 \%$ \\
\hline \multicolumn{2}{|c|}{ Distal lateral } & 12 & $38.71 \%$ \\
\hline \multicolumn{2}{|c|}{ Distal medial } & 14 & $45.16 \%$ \\
\hline \multicolumn{2}{|c|}{ No wedge } & 6 & $19.35 \%$ \\
\hline \multicolumn{2}{|c|}{ Autograft (medial) } & 1 & $3.23 \%$ \\
\hline Tibia & & Number of patients & $\%$ \\
\hline Medial & & 23 & $74.19 \%$ \\
\hline Lateral & & 12 & $38.71 \%$ \\
\hline \multicolumn{2}{|c|}{ No wedge } & 7 & $22.58 \%$ \\
\hline \multicolumn{2}{|c|}{ Autograft (lateral) } & 1 & $3.23 \%$ \\
\hline
\end{tabular}

Table 2 - Mean number of wedges and size of the polyethylene.

\begin{tabular}{c|c|c|c}
\hline Group & $\begin{array}{c}\text { Number of } \\
\text { knees }\end{array}$ & $\begin{array}{c}\text { Number of wedges } \\
\text { (mean) }\end{array}$ & Polyethylene (mean) mm \\
\hline I & 6 & 1.33 & 16.17 \\
\hline IIA & 7 & 2 & 12 \\
\hline IIB & 12 & 4.33 & 12.83 \\
\hline III & 6 & 4.83 & 14.17 \\
\hline
\end{tabular}

A statistically significant difference was found between the groups, with an increasing need for the use of wedges between groups I and III. For statistical analysis of the results obtained, the statistical Kruskal-Wallis test was used $(\mathrm{P}=0.0012)$. No statistically significant difference was found between the groups in relation to the thickness of the polyethylene used.

In $66.6 \%$ of the patients of group I and $42.8 \%$ of the patients in group IIA, it was necessary to use wedges in sites that were not planned prior to surgery (demonstrating an error in the preoperative classification). All were $5 \mathrm{~mm}$, and they were placed in the following sites: distal-lateral femur $(23 \%)$, distal-medial femur (23\%), posterolateral femur 
(15.4\%), posteromedial femur (15.4\%) and medial tibia $(7.7 \%)$. The femur was largely responsible for the planning error in the identification of bone failures through the preoperative radiographs.

Of the patients in group IIA, $85.7 \%$ presented defects in the medial tibial plateau in the preoperative radiographs, and $28.5 \%$ presented defects in the medial femoral condyle. After the revision surgery, all these patients required wedges in these sites, according to the diagnosis carried out before the surgery.

\section{DISCUSSION}

The increase in the number of total knee arthroplasties carried out ${ }^{(1,2)}$ will probably lead to an increase in the number of revisions of these arthroplasties. The difficulties encountered in revisions are varied, and should be minimized. Correct preoperative planning in review total knee arthroplasties is essential, and involves planning the need for wedges and/or structured $\operatorname{grafts}^{(10,11,23,24)}$.

Preoperative assessment of the revision surgeries through magnetic resonance imaging or computed tomography is greatly hampered by the presence of the implants, which generate large artifacts on the image. A simple, low-cost solution is the use of radiographs.

An adequate standardization of the bone defects is obtained through the AORI classification ${ }^{(20,21)}$. A new challenge, therefore, is to associate the preoperative radiographs with the intraoperative findings.

The AORI classification for bone defects of the knee based on preoperative radiographs showed a growing correlation with the need for the use of wedges and/or structured grafts in revision total knee arthroplasty. The use of wedges, bone graft or polyethylene thickness to compensate for defects was left to the discretion of each individual surgeon, since the objective was not to assess the methods of bone filling. The knees in group I used, on average, 1.33 wedges, and this number gradually increased to an average of 4.83 wedges for the knees of group III. There was also a correct correlation between the location of the defects and the localization of the wedges used, and the AORI classification proved useful for preoperative planning in these patients.

Also, caution is needed with the femoral assessment in the radiographs. In $46 \%$ of the knees of groups I and IIA, small bone gaps (up to $5 \mathrm{~mm}$ ) were not diagnosed; therefore, the use of implants without the option of wedges in these patients should be done very carefully. The high number of posterolateral and posteromedial femoral defects found is also emphasized, and the greater difficulty in assessing the presence of these defects due to the overlapping of the condyles in the metal implants.

The average polyethylene thickness did not present significant differences among the various groups studied (I, IIA, IIB and III). The use of a thicker polyethylene in some cases, or tibial blocks, did not alter the joint interline given by the height of the femoral component; thus, the increase of the thickness of the polyethylene was an option for symmetrical defects in both tibial plateaux, and the small variations that did not demonstrate statistical correlation can be classified as merely descriptive. The correct filling of bone defects with wedges, cement or grafts enables correction of the ligament balance, although this is not the objective of this study, through the use of a thicker polyethylene in some cases; therefore, the absence of differences between the groups, in terms of polyethylene thickness, does not invalidate the AORI classification

In this initial analysis, the pre- and postoperative functional results were not discussed; therefore, new studies should be carried out to determine whether there is a correlation between the AORI classification and the functional prognosis of patients submitted to revision total knee arthroplasty.

\section{CONCLUSION}

The AORI classification for bone defects in the knee based on preoperative radiographies showed an increasing correlation with the need for the use of wedges and/or structured grafts in revision total knee arthroplasty; however, up to $46 \%$ of the knees in groups I and IIA presented bone gaps of up to $5 \mathrm{~mm}$ that were undiagnosed in the preoperative radiographs, demonstrating that this classification is not capable of fully anticipating the intraoperative findings. 


\section{REFERENCES}

1. Khatod M, Inacio M, Paxton EW, Bini SA, Namba RS, Burchette RJ, et al. Knee replacement: epidemiology, outcomes, and trends in Southern California: 17,080 replacements from 1995 through 2004. Acta Orthop. 2008;79(6):812-9.

2. Wells VM, Hearn TC, McCaul KA, Anderton SM, Wigg AE, Graves SE. Changing incidence of primary total hip arthroplasty and total knee arthroplasty for primary osteoarthritis. J Arthroplasty. 2002;17(3):267-73.

3. Järvenpää J, Kettunen J, Miettinen $H$, Kröger $H$. The clinical outcome of revision knee replacement after unicompartmental knee arthroplasty versus primary total knee arthroplasty: 8-17 years follow-up study of 49 patients. Int Orthop. 2010;34(5):649-53.

4. Friedman RJ, Hirst P, Poss R, Kelley K, Sledge CB. Results of revision total knee arthroplasty performed for aseptic loosening. Clin Orthop Relat Res. 1990;(255):235-41.

5. Brand MG, Daley RJ, Ewald FC, Scott RD. Tibial tray augmentation with modular metal wedges for tibial bone stock deficiency. Clin Orthop Relat Res. 1989;(248):71-9.

6. Conditt MA, Parsley BS, Alexander JW, Doherty SD, Noble PC. The optimal strategy for stable tibial fixation in revision total knee arthroplasty. J Arthroplasty. 2004;19(7 Suppl 2):113-8.

7. Cuckler JM. Bone loss in total knee arthroplasty: graft augment and options. J Arthroplasty. 2004;19(4 Suppl 1):56-8.

8. Dorr LD. Bone grafts for bone loss with total knee replacement. Orthop Clin North Am. 1989;20(2):179-87.

9. Engh GA, Ammeen DJ. Use of structural allograft in revision total knee arthroplasty in knees with severe tibial bone loss. J Bone Joint Surg Am. 2007;89(12):2640-7.

10. Hockman DE, Ammeen D, Engh GA. Augments and allografts in revision total knee arthroplasty: usage and outcome using one modular revision prosthesis. J Arthroplasty. 2005;20(1):35-41

11. Pagnano MW, Trousdale RT, Rand JA. Tibial wedge augmentation for boné deficiency in total knee arthroplasty. A followup study. Clin Orthop Relat Res.1995;(321):151-5.
12. Radnay CS, Scuderi GR. Management of bone loss: augments, cones, offset stems. Clin Orthop Relat Res. 2006;446:83-92.

13. Rand JA. Bone deficiency in total knee arthroplasty. Use of metal wedge augmentation. Clin Orthop Relat Res. 1991;(271):63-71.

14. Rand JA. Modular augments in revision total knee arthroplasty. Orthop Clin North Am. 1998;29(2):347-53.

15. Werle JR, Goodman SB, Imrie SN. Revision total knee arthroplasty using large distal femoral augments for severe metaphyseal bone deficiency: a preliminary study. Orthopedics. 2002;25(3):325-7.

16. Deehan DJ, Murray J, Birdsall PD, Holland JP, Pinder IM. The role of the rotating hinge prosthesis in the salvage arthroplasty setting. J Arthroplasty. 2008;23(5):683-8

17. Joshi $\mathrm{N}$, Navarro-Quilis $\mathrm{A}$. Is there a place for rotating-hinge arthroplasty in knee revision surgery for aseptic loosening? J Arthroplasty. 2008;23(8):1204-11.

18. Pour AE, Parvizi J, Slenker N, Purtill JJ, Sharkey PF. Rotating hinged total knee replacement: use with caution. J Bone Joint Surg Am. 2007;89(8):1735-41.

19. Insall JN. Revision of aseptic failed total knee arthroplasty. In: Insall JN, editor. Surgery of the knee. New York: Churchill Livingstone; 1993. p. 935-57.

20. Engh GA. A classification of bone defects. In: Engh GA, editor. Revision knee arthroplasty. Baltimore: Williams \& Wilkins; 1997. p. 63-120.

21. Engh GA. Classification of bone defects femur and tibia. In: Scuderi GR, Tria AJ, editor. Knee arthroplasty handbook. New York: Springer;2006. p. 116-32.

22. Pecora J, Hinckel BB, Demange MK, Gobbi RG, Tirico Luis EP, lamaguchi MM. Correlação inter-observador da classificação de falhas ósseas em artroplastia de joelho. Acta Ortop Bras. 2011;19(6):368-72.

23. Clatworthy MG. Management of bony defects in revision total knee arthroplasty. In: Callaghan JJ, editor. The adult knee. Philadelphia: Lippincott Williams \& Wilkins; 2003. p. 1455-64.

24. Pagnano MW. Management of bone defects. In: Insall JN, editor. Surgery of the knee. Philadelphia: Churchill Livingstone/Elsevier; 2006. p.1799-813. 